\title{
Author Correction: Reappraising the spectrum of AKI and hepatorenal syndrome in patients with cirrhosis
}

\author{
Juan Carlos Q. Velez(10, George Therapondos (10) and Luis A. Juncos
}

Nature Reviews Nephrology (2019) https://doi.org/10.1038/s41581-019-0218-4 Published online 13 November 2019

In Table 2 of the original article published online, the response rates achieved with terlipressin and noradrenaline as described by reference 197 were incorrectly stated to be $70 \%$ and $83 \%$, whereas they should have been $83 \%$ and $70 \%$. In addition, a footnote has now been added to Table 2 to indicate studies that included patients with HRS-2. Finally, the overall reversal rates achieved with terlipressin based on studies outside the USA, which were described in the original article to range from $\sim 40-70 \%$, have been corrected to $\sim 40-80 \%$. These corrections have been made to the HTML and PDF versions of the manuscript.

https://doi.org/10.1038/s41581-020-0255-z I Published online 27 January 2020

C Springer Nature Limited 2020 